\title{
Permeability and its Influence on the Broadband Frequency Response of a Power Transformer
}

\author{
Steven D. Mitchell \\ School of Electrical and \\ Computer Engineering \\ University of Newcastle \\ Callaghan, Australia 2308 \\ Email: steve.mitchell@newcastle.edu.au
}

\author{
James S. Welsh \\ School of Electrical and \\ Computer Engineering \\ University of Newcastle \\ Callaghan, Australia 2308 \\ Email: james.welsh@newcastle.edu.au
}

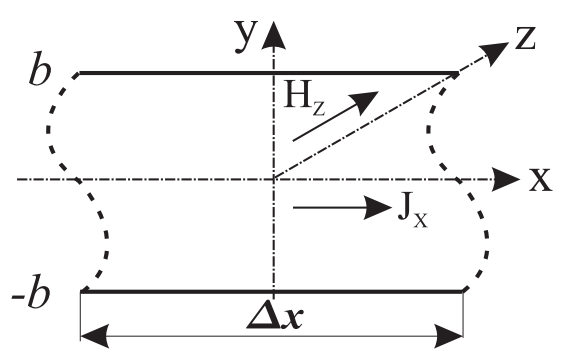

Fig. 1. One dimensional eddy current model

at $1 \mathrm{MHz}$ and approaches unity at frequencies beyond $15 \mathrm{MHz}$. It also demonstrates that there is a degree of independence with regards to the FRA test source voltage due to the low field conditions generated by an FRA swept frequency test.

\section{THEORY}

mechanical and electrical properties. The degree of variation in the frequency response can be used as a figure of merit to determine whether more invasive testing is required [1]. Whilst significant work has been published detailing methods of quantifying the change [2] [3], work in the area of physically interpreting the phenomena behind the change is in need of further attention [4]. The report [4] emphasises the need for FRA research to pay particular attention to the physical modeling of a power transformer.

Work to date on physically based transformer models has typically neglected the influence of the core beyond $50 \mathrm{kHz}$ [5] [6] [7]. A recent paper by Abeywickrama [8] extended the frequency range and stated that the complex permeability of the transformer core was still significant beyond $100 \mathrm{kHz}$ for FRA based testing. However, work by Tavner [9] on the coupling of discharge currents in the laminated steel stator core of electrical machines, demonstrated relative complex permeability greater than unity at $10 \mathrm{MHz}$ for $0.35 \mathrm{~mm}$ laminations. It should be noted that typical lamination thickness used in power transformer manufacture range from $0.23 \mathrm{~mm}$ to $0.35 \mathrm{~mm}$ [10] and as such this work should be similarly applicable to transformers.

This paper demonstrates both theoretically and experimentally that the relative complex permeability is still significant

\section{A. One Dimensional Analysis of Eddy Currents}

The laminations in the core of a transformer have a thickness which is significantly smaller than its width and length. In addition, eddy currents tend to flow close to the conductor surface. As such, the examination of eddy currents in a transformer lamination can be accomplished in an approximate manner by considering current flow in one dimension only (Figure 1). This approximation leads to the derivation of the Diffusion Equation [11]. This is an important relationship which equates the delay and attenuation in the changing magnetic field inside the lamination to the induced eddy currents. Assuming that the excitation is time harmonic [12], then the Diffusion Equation may be written as:

$$
\begin{aligned}
\frac{\partial^{2} H_{z}}{\partial y^{2}} & =j \omega \sigma \mu_{0} \mu_{r} H_{z} \\
& =\gamma^{2} H_{z},
\end{aligned}
$$

where $\gamma=\sqrt{j \omega \sigma \mu_{0} \mu_{r}}$ is the propagation constant. Solving for $H$ (direction now assumed):

$$
H=\beta \cosh (\gamma y) \quad \text { where } \beta=\text { constant } .
$$


With reference to Figure 1, deriving the flux in a cross sectional slice $2 b \Delta_{x}$ we have,

$$
\begin{aligned}
\Phi & =\int_{-b}^{b} \mu_{0} \mu_{r} H \Delta_{x} d y \\
& =\int_{-b}^{b} \mu_{0} \mu_{r} \Delta_{x} \beta \cosh (\gamma y) d y \\
& =\frac{2 \mu_{0} \mu_{r} \Delta_{x} \beta \sinh (\gamma b)}{\gamma} .
\end{aligned}
$$

Solving (3) for $\beta$,

$$
\beta=\frac{\gamma \Phi}{2 \mu_{0} \mu_{r} \Delta_{x} \sinh (\gamma b)} .
$$

Substituting (4) into (2),

$$
H=\frac{\gamma \Phi \cosh (\gamma y)}{2 \mu_{0} \mu_{r} \Delta_{x} \sinh (\gamma b)} .
$$

As the magnetic field intensity will be unattenuated at the surface of the lamination $(y=b)$,

$$
\grave{H}=\frac{\gamma \Phi \cosh (\gamma b)}{2 \mu_{0} \mu_{r} \Delta_{x} \sinh (\gamma b)} .
$$

The space average magnetic flux density $\bar{B}$ in the $\mathrm{z}$ direction relates the flux through the cross sectional area as,

$$
\bar{B}=\frac{\Phi}{2 b \Delta_{x}} .
$$

The relative complex permeability of a lamination is the ratio of the space average flux density to the surface magnetic field intensity. This relationship is then,

$$
\mu_{s}=\frac{1}{\mu_{0}} \cdot \frac{\bar{B}}{\grave{H}}=\frac{\mu_{r}}{\gamma b} \tanh (\gamma b) .
$$

Lamination stacking factors for silicon steel can have values of $0.95-0.98$ [13]. Therefore the complex permeability relationship (8) will approximate the transformer core permeability.

\section{B. Complex Permeability and its Relationship with Winding Impedance}

The inductance of a winding and its associated magnetic losses can be represented as an impedance which incorporates the complex permeability [14],

$$
\begin{aligned}
Z & =j w L+R \\
& =j w L_{0}\left(\mu_{s}^{\prime}-j \mu_{s}^{\prime \prime}\right),
\end{aligned}
$$

where $L=$ Winding inductance

$$
\begin{aligned}
R & =\text { Magnetic loss resistance } \\
L_{0} & =\text { Unity permeability inductance } \\
\mu_{s}^{\prime} & =\operatorname{Re}\left[\mu_{s}\right] \\
\mu_{s}^{\prime \prime} & =\operatorname{Im}\left[\mu_{s}\right] .
\end{aligned}
$$

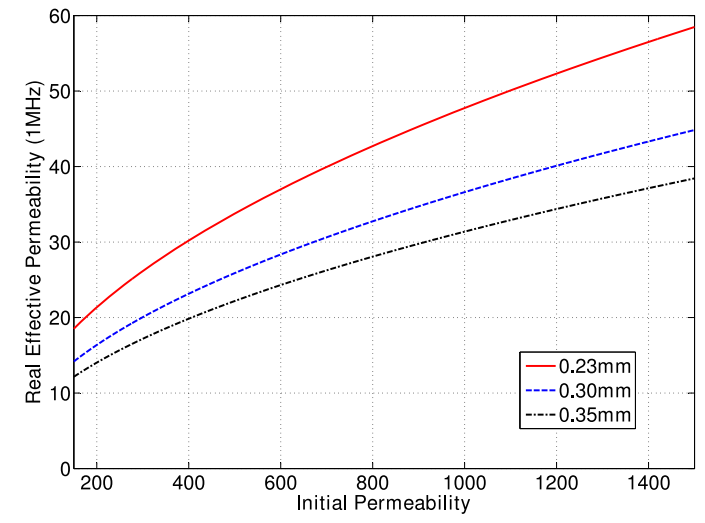

Fig. 2. Real component of the theoretically calculated relative complex permeability at $1 \mathrm{MHz}$ versus the initial permeability for lamination thickness's of $0.23 \mathrm{~mm}, 0.30 \mathrm{~mm}$ and $0.35 \mathrm{~mm}$

\section{Low Field Strength Relative Permeability}

Frequency Response Analysis (FRA) involves the injection of a swept sine wave or impulse signal in order to determine the frequency response of the device under test. The amplitude of the injection signal voltage is generally many orders of magnitude smaller than the designed operating voltage for the transformer. Examples of the injection voltages used in practice are the Doble M5400 which injects $7 V_{R M S}(50 \mathrm{ohm}$ load) and the HP89410A which has a test range up to a maximum of $2.2 V_{R M S}$. These voltages even at mains frequency will result in an induced magnetic field well below the operating "knee" of the B-H hysteresis curve for the core material. As such, these ac signals are considered to be low field. In addition, with increased frequency there will be a resulting increase in the inductive impedance and also in the influence of parasitic winding capacitance. This will correspond to a further decrease in the inductor test current and hence the associated magnetic field intensity. A change in the magnetic field intensity tends to result in a change in the relative permeability due to the nonlinear hysteresis curve. However, under low field conditions, the relative permeability $\left(\mu_{r}\right)$ will approach a value equal to that of the initial permeability $\left(\mu_{i}\right)[15]$,

$$
\mu_{r} \approx \mu_{i}=\frac{1}{\mu_{0}} \lim _{H \rightarrow 0}\left[\frac{B}{H}\right] .
$$

The relationships derived in (8) and (10) can now be rewritten for the low field testing conditions associated with FRA,

$$
\mu_{s}=\mu_{s}^{\prime}-j \mu_{s}^{\prime \prime} \approx \frac{\mu_{i}}{\gamma b} \tanh (\gamma b) .
$$

\section{RESULTS}

\section{A. Theoretical Estimation of the High Frequency Complex Permeability for a Power Transformer}

The purpose of this paper is to demonstrate that the complex permeability of the core of a power transformer remains significant for the entire FRA spectrum. A plot of the real 
TABLE I

INITIAL PERMEABILITY FOR VARIOUS GRADES OF ELECTRICAL STEEL. DATA FROM [15]

\begin{tabular}{|c|c|}
\hline Electrical Steel Grade & $\mu_{i}$ \\
\hline \hline BSI A.253, Ferrosil 253, Losil 25 & 150 \\
\hline Magnesil & $300-1100$ \\
\hline BSI C.86, Ferrosil 86, Transil 86 & 550 \\
\hline BSI grade 62, Alphasil 62, Unisil 62 & 1000 \\
\hline Silectron, Hypersil, Transcor 3X & 1500 \\
\hline
\end{tabular}

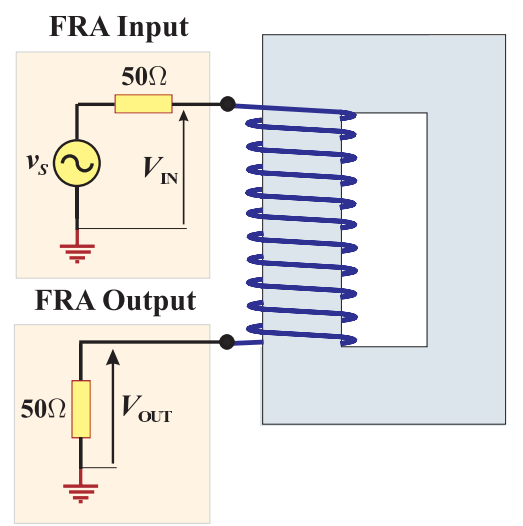

Fig. 3. FRA test bed. Laminated core length $=900 \mathrm{~mm}$, core dimension $=32 \times 37 \mathrm{~mm}$. For frequencies $<1 \mathrm{MHz}:$ Instrument $=\mathrm{HP} 89410 \mathrm{~A}$ Vector Analyser, Number of turns $=200$, winding length $=140 \mathrm{~mm}$, winding diameter $=90 \mathrm{~mm}$. For frequencies $\geq 1 \mathrm{MHz}:$ Instrument $=$ Agilent E5071B Network Analyser, Number of turns $=5$, winding length $=4 \mathrm{~mm}$, winding diameter $=$ $90 \mathrm{~mm}$.

component of the theoretically calculated relative complex permeability at $1 \mathrm{MHz}$ versus initial permeability is provided in Figure 2 via the relationship (12). The Initial Permeability range is based on typical values taken from Table I. The lamination thickness for a power transformer generally ranges between $0.23 \mathrm{~mm}$ and $0.35 \mathrm{~mm}$ [10]. A conductivity of $\sigma=2.1$ x $10^{6} \mathrm{~S} / \mathrm{m}$ (Steel grades - Unisil, M-2 through M-6, JIS: 30P105) was used since grain oriented silicon steel is the predominant form used in transformer core manufacture [8].

The calculated minimum for the real component of the relative complex permeability is an order of magnitude above unity at $1 \mathrm{MHz}$ in Figure 2. This implies that coupling between windings on different phases must be considered when modeling the transformer for high frequency analysis.

\section{B. FRA High Frequency Test Bed}

A single layer inductor has no interlayer and little interturn capacitance. As such, it does not possess the low frequency self resonance of multilayer windings. If the distributed, winding to ground, shunt capacitance is kept to a minimum, it is possible to obtain a frequency response that is completely dominated by the inductive response for frequencies greater than $1 \mathrm{MHz}$. This inductive response over a large range of frequencies provides an ideal test bed to visually observe the effects of complex permeability. The test bed winding will experience similar low field conditions experienced by a power transformer during an FRA test. Figure 3 depicts the test setup used for the following experiments.
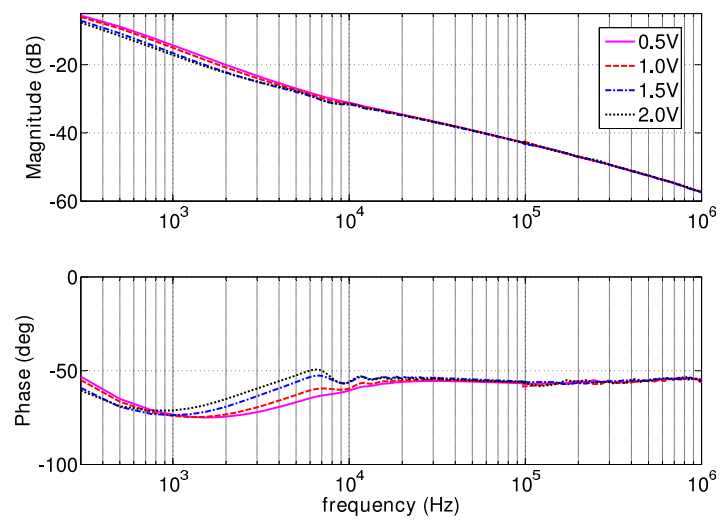

Fig. 4. Frequency response of single layer 200 turn laminated core inductor with various test voltages $\left(0.5 V_{R M S}, 1.0 V_{R M S}, 1.5 V_{R M S}, 2.0 V_{R M S}\right.$
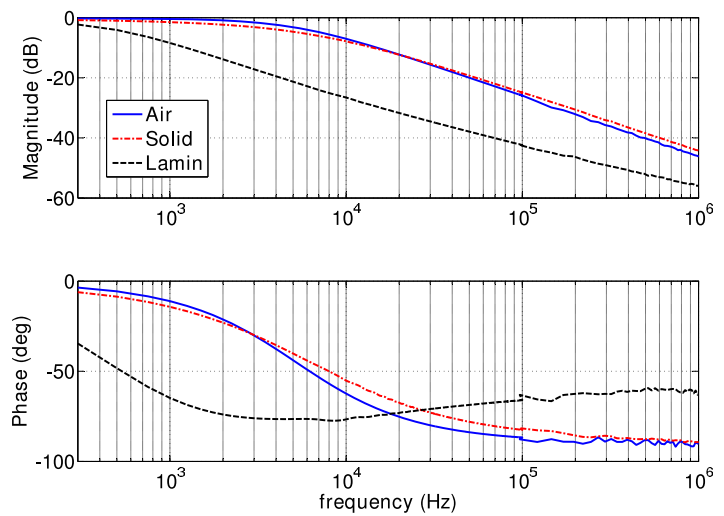

Fig. 5. Broadband frequency response of a 200 turn single layer inductor with a) Air core, b) Solid steel core and c) Laminated electrical steel core (0.35mm laminations)

\section{Practical Confirmation of the Low Field Assumption}

In the theoretical section II-C, the conjecture was made that the FRA injection voltage would generate a magnetic field intensity that could be considered as a low field over the FRA frequency spectrum $(<10 \mathrm{MHz})$. The assumption was made that the relative permeability will approximate an initial permeability and remain relatively constant for various test voltages and the test frequency spectra. Figure 4 demonstrates that this approximation is in fact valid. The observed frequency response for each test voltage has only minor variations at frequencies less than $10 \mathrm{kHz}$ and can be considered identical above $10 \mathrm{kHz}$.

\section{Practical Demonstration of High Frequency Complex Per- meability}

To confirm the theoretical results, it is possible to demonstrate, in a practical sense, that the effective complex permeability is significant beyond $1 \mathrm{MHz}$. An FRA test was conducted on a 200 turn single layer inductor with a core 


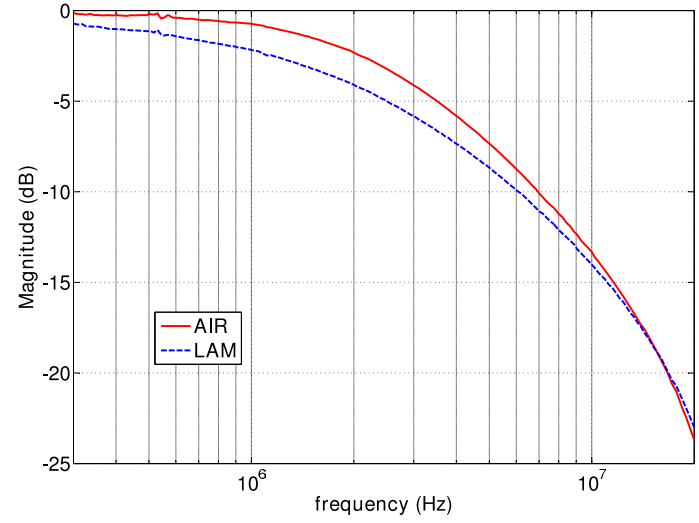

Fig. 6. High frequency FRA test on a 5 turn single layer inductor with a) Air core, b) Laminated $0.35 \mathrm{~mm}$ electrical steel core

constructed from $0.35 \mathrm{~mm}$ silicon steel laminations. To provide a unity permeability reference, an identical test was also conducted on the winding in an air cored configuration. To complete the test series, a test on the winding with a solid steel core was conducted to demonstrate the effect of the relative complex permeability tending to unity. The results are presented in Figure 5.

The frequency responses in Figure 5 validate the theoretical results discussed in the previous section. The laminated core configuration has significantly more attenuation than the air cored version beyond $1 \mathrm{MHz}$. This is indicative of a larger inductance which, since the same winding is used for all tests, corresponds to a permeability larger than one. In fact, modeling tests which utilised the relationship (10) were conducted on the laminated core frequency response, and an estimated relative complex permeability of $\mu_{s}=36-36 j$ was found. The phase response of the laminated core configuration has an upward trend in phase, with respect to frequency, as $\mu_{s}^{\prime \prime} \Rightarrow \mu_{s}^{\prime}$ with the increasing influence of magnetic skin effect and its associated eddy current losses.

The winding with the solid steel core demonstrates the full impact of magnetic skin effect. Within a few kilohertz, the core dimensions exceed the skin depth $\left(\delta=\sqrt{2 / w \sigma \mu_{0} \mu_{r}}\right)$ with the relative complex permeability tending to unity and approximately tracking the air cored winding frequency response.

\section{E. A Relative Complex Permeability of Unity}

At frequencies beyond a couple of megahertz, the measurement equipment itself begins to become influential in the observed FRA test. The measurement cables will have distributed capacitance of $100 \mathrm{pF} / \mathrm{m}$ and therefore the usefulness of FRA testing beyond a couple of megahertz becomes questionable. The recommended diagnostic range for a M5400 SFRA test device is $20 \mathrm{~Hz}-2 \mathrm{MHz}$ [16]. However, in order for the investigation to be rigorous, work was conducted to locate the frequency at which the relative complex permeability for a $0.35 \mathrm{~mm}$ laminated silicon steel core is approximately unity.
In order to achieve this without self resonant interference and to minimise transmission line effects, a winding with only five turns was used. The test was conducted using an Agilent E5071B Network Analyser in the same configuration as Figure 3. Neglecting the influence of parasitic elements in the observed frequency response, the results in Figure 6 quantitively demonstrate that the laminated core winding has a larger inductive impedance than the corresponding air cored winding, at frequencies greater than $15 \mathrm{MHz}$. As all the other parameters are equal, the relative complex permeability must also be above unity until frequencies beyond $15 \mathrm{MHz}$. It should also be noted that this frequency would only be higher for the smaller lamination thickness values used in power transformer manufacture.

\section{CONCLUSION}

This paper demonstrates how the complex permeability for a power transformer remains significant for frequencies above $1 \mathrm{MHz}$. In fact, the relative complex permeability will not approach unity till frequencies larger than $15 \mathrm{MHz}$. The paper has also demonstrated that the frequency response is relatively independent of the FRA test voltage despite the highly nonlinear nature of the hysteresis curve.

\section{REFERENCES}

[1] C. L. Sweetser, "Experience with sweep frequency response analysis (sfra) measurements," Doble Engineering Company, Tech. Rep.

[2] S. Ryder, "Methods for comparing frequency response analysis measurements," Electrical Insulation, 2002. Conference Record of the 2002 IEEE International Symposium on, pp. 187-190, Apr 2002.

[3] J. Welsh, C. Rojas, and S. Mitchell, "Wideband parametric identification of a power transformer," Universities Power Engineering Conference, 2007. AUPEC 2007. Australasian, pp. 1-6, Dec. 2007.

[4] "Mechanical condition assessment of transformer windings using frequency response analysis (fra)," ELECTRA - CIGRE WG A2.26 Report, vol. 228, October 2006.

[5] Z. Wang, P. Crossley, and K. Cornick, "A simulation model for propagation of partial discharge pulses in transformers," Power System Technology, 1998. Proceedings. POWERCON '98. 1998 International Conference on, vol. 1, pp. 151-155 vol.1, Aug 1998.

[6] G. Gharehpetian, H. Mohseni, and K. Moller, "Hybrid modelling of inhomogeneous transformer winding for very fast transient overvoltage studies," Power Delivery, IEEE Transactions on, vol. 13, no. 1, Jan 1998.

[7] E. Rahimpour, J. Christian, K. Feser, and H. Mohseni, "Transfer function method to diagnose axial displacement and radial deformation of transformer windings," Power Delivery, IEEE Transactions on, vol. 18, no. 2, pp. 493-505, April 2003.

[8] K. Abeywickrama, T. Daszczynski, Y. Serdyuk, and S. Gubanski, "Determination of complex permeability of silicon steel for use in high-frequency modeling of power transformers," Magnetics, IEEE Transactions on, vol. 44, no. 4, pp. 438-444, April 2008.

[9] P. Tavner and R. Jackson, "Coupling of discharge currents between conductors of electrical machines owing to laminated steel core," Electric Power Applications, IEE Proceedings B, vol. 135, no. 6, Nov 1988.

[10] S. V. Kulkarni and S. A. Khaparde, Transformer Engineering: Design and Practice. Marcel Dekker, 2004.

[11] R. L. Stoll, The Analysis of Eddy Currents. Clarendon Press, 1974.

[12] D. K. Cheng, Field and Wave Electromagnetics. Addision-Wesley, 1983.

[13] M. J. Heathcote, The $J \&$ P Transformer Book, twelfth ed. Reed Educational and Professional Publishing Ltd, 1998.

[14] E. C. Snelling, Soft Ferrites. Iliffe Books Ltd, 1969.

[15] K. L. Kaiser, Electromagnetic Compatibility Handbook. CRC Press, 2004.

[16] "M5400 sweep frequency response analyser - technical specification," Doble Engineering Company, Tech. Rep. 